\title{
APROXIMACIONES A LOS USOS DE TWITTER EN BIBLIOTECAS UNIVERSITARIAS DE ARGENTINA
}

\author{
Claudia Nora Laudano* \\ Instituto de Investigaciones en Humanidades y Ciencias Sociales (UNLP-CONICET). Facultad de Humanidades y Ciencias de la \\ Educación (FaHCE). Universidad Nacional de La Plata (UNLP). \\ Javier Planas ** \\ Instituto de Investigaciones en Humanidades y Ciencias Sociales (UNLP-CONICET). Facultad de Humanidades y Ciencias de la \\ Educación (FaHCE). Universidad Nacional de La Plata (UNLP). \\ María Inés Kessler ** \\ Instituto de Investigaciones en Humanidades y Ciencias Sociales (UNLP-CONICET). Facultad de Humanidades y Ciencias de la \\ Educación (FaHCE). Universidad Nacional de La Plata (UNLP)
}

\begin{abstract}
Resumen: El artículo analiza los principales usos de la plataforma Twitter por parte de bibliotecas universitarias en Argentina. Luego de la revisión bibliográfica, se exponen los procedimientos metodológicos empleados para identificar las instituciones que cuentan con esta herramienta comunicacional en la actualidad y los usos que se hace de ella a partir de ítems tales como: datos institucionales básicos, visibilidad y accesibilidad de la cuenta de Twitter en la web de la biblioteca, momento de inicio de la actividad, volumen histórico de tuits, seguidores y siguiendo, así como la cantidad y el tipo de publicaciones realizadas en el período del relevamiento de datos. En los resultados se observa que un escaso número de bibliotecas adoptaron Twitter y que los usos de la herramienta muestran en general utilizaciones no planificadas, más centradas en la difusión que la interacción con las y los usuarios. Asimismo, se destaca que pese a existir una abundante bibliografia sobre lo que se consideran las buenas prácticas, en general no son adoptadas por las instituciones. Finalmente, se proponen estudios complementarios para conocer rutinas laborales de los bibliotecarios al respecto.
\end{abstract}

Palabras clave: redes sociales; Twitter; bibliotecas universitarias; comunicación; Argentina.

Title: APPROXIMATIONS TO THE USES OF TWITTER BY UNIVERSITY LIBRARIES IN ARGENTINA.

Abstract: This article analyses the main uses of the social media Twitter in university libraries in Argentina. After revising existing literature, we outline the research methods used to identify whether libraries are currently adopting Twitter and how it is being used. We focus on the following areas: the starting date of the activity, basic institutional data, visibility and access to Twitter from the library web, the quantity of tweets over time, those "followed" and those "following" and quantity and type of posts during the period of time selected for research. The results show that few libraries have used Twitter and their use of this media tool has generally been unplanned, mostly for spreading information rather than interaction. It also stresses that despite an extensive literature on what are considered good practices, generally they are not taken by the institutions. At last, we propose further research in this area to widen our knowledge of the daily use of Twitter among librarians.

Keywords: social media; Twitter; university libraries; communication; Argentina.

\section{INTRODUCCIÓN}

Entre las transformaciones más visibles en las bibliotecas, vinculadas con las tecnologías de la comunicación y la información en las últimas décadas, se registra un paulatino abandono de medios impresos como instancias privilegiadas de contacto con los usuarios, o, en su defecto, la persistencia de éstos en situación minoritaria junto a un menú considerable de medios digitales. Desde mediados de la primera década del siglo XXI, las denominadas redes sociales lograron instalarse en las prácticas cotidianas de millones de personas en diferentes partes del planeta. Entre las más conocidas y utilizadas en el territorio argentino se encuentran Facebook, Youtube y Twitter, con cifras estimadas (no oficiales) que rondarían los 25 millones de usuarios mensuales para la primera y 6 millones para la última, en mayor medida desde conexiones móviles. Según fuentes periodísticas y cálculos de la propia empresa, el uso de Twitter ha mostrado un crecimiento notable en los últimos años, un incremento que parece explicarse en las apelaciones constantes que los programas de radio y televisión hacen de la red social, además de la fluida relación que mantienen con este medio la farándula, los deportistas y los políticos.

\footnotetext{
*claudialaudano@gmail.com

*** planasjavier@yahoo.com.ar

*** kessler.mines@gmail.com
}

Recibido: 16-12-2015; 2 $2^{\mathrm{a}}$ versión: 11-07-2016; aceptado: 19-07-2016.

LAUDANO, C.N.; PLANAS, J. y KESSLER, M.I. Aproximaciones a los usos de Twitter en bibliotecas universitarias de Argentina. Anales de Documentación, 2016, vol. 19, nº 2. Disponible en: http://dx.doi.org/10.6018/analesdoc.19.2.246291. 
Luego de analizar en sendas investigaciones los usos de Facebook por parte de bibliotecas académicas y universitarias de Argentina (Laudano et al. 2014, 2015), en esta oportunidad interesa relevar y analizar lo concerniente a las utilizaciones de Twitter en las bibliotecas universitarias. En tal sentido, cabe recordar que Twitter es una plataforma digital que permite enviar mensajes de 140 caracteres, está en funcionamiento desde 2006 y alcanzó un pico de difusión en 2009. En tiempos de predominio audiovisual, se benefició con la posibilidad de incluir envíos de imágenes y vídeos junto a los textos breves.

\section{ANTECEDENTES}

Desde el lanzamiento de Twitter como plataforma de microbbloging, el campo bibliotecológico registra un interés creciente por su empleo para los objetivos específicos. A la vez, como sugiere Margaix Arnal (2008), los profesionales procuran estar donde sus usuarios están respecto de los recursos digitales y utilizar sus mismos canales de comunicación. En tal sentido, parte de la literatura académica disponible se basa en la realización de relevamientos periódicos de los usos y las aplicaciones de Twitter en bibliotecas de diferente tipología, algunos con énfasis comparativos (Aharony, 2010, Del Bosque, Leif y Skarl, 2012; Chen, Chu y Xu, 2012; Verishagen y Hank, 2014; Cavanagh, 2015; entre otros).

Según la revisión bibliográfica específica realizada por Sewell (2015), la mayoría de los trabajos centraliza la mirada en aspectos concernientes a la difusión de información; mientras que un conjunto de ellos analiza los principales focos de interés de los tuits (Stuart, 2010). Al respecto, para perfilar acerca de qué tuitean, los trabajos de investigación retornaron a estudios basados en análisis de contenido, una perspectiva clásica en el campo de las ciencias sociales, que aporta un estado descriptivo de las categorías en uso en determinados textos, sin analizar sus significaciones en diferentes contextos.

Entre ellos, a modo de ejemplos, Aharony (2010), mediante un estudio de 30 bibliotecas estadounidenses, públicas y académicas, durante tres meses, indicó que ambos tipos de instituciones coinciden en tuitear, en orden decreciente, en torno a las categorías "información acerca de la biblioteca", "misceláneas" y "tecnología". Por su parte, Stvilia y Gibradze (2014) realizaron un estudio exploratorio con 752 tuits de las cuentas de 6 bibliotecas académicas de universidades públicas estadounidenses, en los que identificaron nueve tipos de tuits. De ellos, 'eventos', 'recursos' y 'construcción de comunidad', figuran entre las categorías con ocurrencias más frecuentes.

En cuanto a los servicios de referencia, Mathews (2008) planteó tempranamente que podían contemplarse instancias informales o casuales de conversación en Twitter para sugerir recursos o servicios bibliotecarios. Estudios posteriores ofrecen panoramas diferentes acerca del desarrollo del potencial de la herramienta en servicios de referencia. En tal sentido, Fields (2010) propuso un estado breve de cómo el personal bibliotecario utiliza Twitter en particular para potenciar el servicio de referencia desde las cuentas institucionales en varias universidades estadounidenses; mientras que Del Bosque, Leif y Skarl (2012), tras analizar los usos de Twitter en un conjunto de bibliotecas académicas de EE.UU. durante un mes, consideraron que el servicio de referencia era subutilizado.

Hasta el momento, escasos trabajos focalizan en la dimensión "seguidores de cuentas" de las bibliotecas. Entre los estudios de casos, Sewell (2013) analizó un listado de 432 cuentas de seguidores de una biblioteca de ciencias médicas en EE.UU. y, tras construir una tipología, determinó que los principales grupos se hallaban, en orden de importancia, estudiantes, graduados, profesionales, staff, otras bibliotecas y bibliotecarios. En Canadá, tras un minucioso análisis, Cavanagh (2015) registró que entre el grupo mayoritario de seguidores de una biblioteca pública se encontraban miembros locales de la comunidad tanto como empresas. La construcción de estos perfiles facilita a las bibliotecas el desarrollo de estrategias concretas para aumentar y/o consolidar la llegada a sus audiencias predominantes o plantearse nuevos desafíos, sobre la base de información confiable.

Ante cierto auge de estudios métricos en esta área, Waddell, Barnes y Khan-Kernahan (2012) sugieren probar y adaptar instancias de medición acorde a las necesidades de las bibliotecas. En particular, distinguen la posible "exposición" de los tuits, en tanto capacidad de ser vistos por el conjunto de seguidores de las cuentas, del "alcance", como posibilidad de acción promovida por un tuit, como puede ser el retuiteo. Aclaran luego que sólo el "alcance" puede ser medido, por ejemplo, desde los retuiteos o por el inicio de seguimiento a las cuentas.

Respecto de los antecedentes vinculados con estudios en Argentina, De Volder, González Terán y Gutiérrez (2012) analizaron los perfiles y el uso de Twitter por parte de 15 bibliotecas universitarias del país en 2012, mediante la observación de las cuentas y una encuesta. La mayoría de las bibliotecas comenzó a utilizar la plataforma entre 2010 (8/15) y 2011 (4/15). En su trabajo consideraron: cantidad de tuits, cantidad de seguidores, tipo de información que 
difunden, periodicidad de actualización, tiempo dedicado a la gestión de la cuenta, área de la biblioteca que gestiona la cuenta, razones de uso, usuarios, política del servicio y fuentes de contenido. Determinaron que la mayoría de las bibliotecas mantiene el servicio con dos personas, desde las áreas de circulación, referencia y difusión, dedicando a la tarea desde menos de media hora hasta más de una hora al día. El uso predominante de las cuentas es la difusión de información. En otro orden, el 53\% de las bibliotecas tiene una política definida sobre el servicio y 9 de las bibliotecas estudiadas enlazan al perfil de Twitter desde su sitio web. Respecto del empleo de la herramienta notaron que el porcentaje de bibliotecas que la utilizaba era extremadamente bajo.

Sobre la base de este mismo estudio, De Franceschi (2014), a través de un seguimiento trimestral, localizó sólo 10 cuentas en uso. En su trabajo describe las actividades que realizan en Twitter las bibliotecas universitarias argentinas analizando los tipos de tuits: de difusión propia, de redifusión de información, de extensión cultural, de servicio de recomendación e interacción con el usuario. En 9 de las bibliotecas se notó un incremento en el uso del servicio en el período estudiado. Respecto al uso que las bibliotecas hacían de Twitter, en mayor medida fue la redifusión de información y en menor grado la generación propia de contenido, tomando como referencia la información institucional, de perfiles de alumnos y docentes y de medios de comunicación. El servicio de recomendación fue casi nulo, y la interacción con los usuarios muy baja. Concluyó que las bibliotecas universitarias usaban este servicio con una tendencia notable a trabajar la noticia emergente.

En otro orden de cosas, tras años de puesta en práctica de la plataforma para fines bibliotecarios, la literatura académica, en consonancia con la de carácter profesional, abunda en recomendaciones para un uso adecuado y provechoso de la herramienta (Stuart, 2010; Alvim, 2010; Forrestal, 2011; Bodnar y Doshi, 2011; Hagman, 2012; Del Bosque, Leif y Skarl, 2012; Waddell, Barnes y Khan-Kernahan, 2012, entre otros). Dadas las diferentes aristas que abordan, se podrían agrupar en las siguientes dimensiones. En primer lugar, refieren a planificar el potencial que la herramienta puede aportar al desarrollo de las actividades de la biblioteca. En tal sentido, sugieren reflexionar acerca de la finalidad que se proyecta con el uso de la plataforma así como contemplar si es el medio de comunicación más utilizado por el público usuario de la institución o el más pertinente para los fines propuestos.

En otros trabajos se percibe la preocupación por la dedicación temporal y de personal que insume un adecuado sostenimiento de la cuenta, ya que un uso superficial o sin la dedicación necesaria podría perjudicar la imagen institucional y la confianza en la biblioteca. En caso de utilizar más de una cuenta, se sugiere coordinar las actividades. Respecto de la cuenta, se recomienda que la apariencia esté acorde a la imagen institucional general (en el uso del color, logo, fotografía, etc.) y que figure el enlace hacia la página web u otro sitio institucional. Por otro lado, diferentes textos insisten en destacar la importancia de no quedarse en el mero retuiteo o el agradecimiento a otros por ser retuiteado, sino recabar información de necesidades e intereses de seguidores de la cuenta, mediante la utilización de una doble vía comunicacional, más que un uso instrumental de difusión informativa. Asimismo, dentro del conjunto de directrices para ampliar las potencialidades de la herramienta sugieren usar la función de los mensajes directos, arrobar a seguidores, disponer de listas de envíos y/o construir hashtags.

Respecto de los temas pendientes para investigar, entre otros, figuran la necesidad de reflexionar acerca de la construcción de la voz pública de la biblioteca, los alcances de las controversias en línea por el potencial de la viralización, las posibilidades del trabajo en red mediante esta herramienta (y/o junto a otras), tanto como la construcción de comunidad.

Al considerar el estado del campo, se detecta un vacío bibliográfico importante en lo que respecta a los usos de Twitter por parte de las bibliotecas en Argentina. Esta misma ausencia obliga a estudiar, como primer paso hacia trabajos de corte cualitativo, una exploración cuantitativa sobre las maneras en que las bibliotecas utilizan esta herramienta para comunicarse con la comunidad de usuarios. En este sentido, el objetivo primordial de esta investigación es brindar un panorama general de estos empleos y evaluar su rendimiento de acuerdo a los resultados que brindan los análisis realizados en otras latitudes.

\section{METODOLOGÍA}

El estudio, de carácter exploratorio, comprendió el análisis de las bibliotecas universitarias de tres instituciones nacionales: Universidad Nacional de Córdoba (UNC), Universidad de Buenos Aires (UBA) y Universidad Nacional de La Plata (UNLP). Estas universidades públicas fueron seleccionadas por ser las de mayor trayectoria académica en el país: la UNC se fundó en 1613 y se nacionalizó en 1856; la UBA se fundó en 1821 y se nacionalizó en 1881 y la UNLP se fundó en 1905, con su antecedente de la Universidad Provincial de La Plata que funcionaba desde 1897. A través de los sitios web oficiales de cada una se relevaron los de la totalidad de las unidades académicas y, a partir de 
allí, se detectaron los de 43 bibliotecas. Cabe consignar que no se tomaron en consideración las bibliotecas universitarias centrales de la UNC y de la UNLP, dado que los objetivos institucionales de cada una las separan del resto del conjunto.

Para guiar este estudio se retomó la metodología de producción de datos empleada por este equipo de investigación en dos indagaciones precedentes sobre los usos de Facebook de grupo de bibliotecas académicas argentinas (Laudano et al., 2014, 2015). En este sentido, el paso siguiente consistió en constatar la adopción de Twitter por parte de las bibliotecas universitarias a partir del siguiente protocolo de búsqueda: 1) Certificar si en el sitio web de cada entidad se enlazaba la dirección de Twitter. 2) Si estaba disponible, se dejaba constancia de la dirección en una planilla ad hoc. 3) Si no estaba disponible, se continuaba con las siguientes alternativas de rastreo en el buscador de Twitter: 3.1) por el nombre completo de la biblioteca; 3.2) por la denominación de la institución mayor; 3.3) por la sigla. 4) Cuando ninguna de estas opciones arrojaba resultados positivos, se recurrió al buscador de Google empleando los términos anteriores más "Twitter" (Wan, 2011).

Con el listado resultante se elaboró una matriz para volcar la información de interés relativa a los perfiles. Entre los principales ítems recogidos figuran los datos institucionales básicos, la visibilidad y accesibilidad de la cuenta de Twitter en la web de la biblioteca, el año de inicio de la actividad, el volumen histórico de tuits, seguidores y siguiendo, así como también la cantidad y el tipo de publicaciones realizadas entre el 1 y el 26 de junio de 2015 , período de referencia para el relevamiento de la actividad. Esta metodología de producción de datos tuvo como finalidad capturar las dinámicas de publicación que siguen estas instituciones tal y como las perciben sus usuarios.

\section{RESULTADOS}

Sobre las 43 bibliotecas universitarias estudiadas solo 12 utilizan Twitter. No obstante, durante el período en que se efectuó la recolección de datos algunas cuentas no registraron actividad (del 1 al 26 de junio de 2015). Este aspecto, si bien no se consideró suficiente para juzgarlas como desactivadas — pues antes y después se observan tuits—, brinda una idea de la escasa atención que algunas instituciones le prestan. El asunto se revela fundamental si se considera que las recomendaciones para el empleo de esta herramienta sugieren un ritmo fluido de publicación. En cuanto al porcentaje global, un dato interesante emerge al comprobar que estas cuentas pertenecen a bibliotecas que en su mayoría utilizan Facebook como herramienta de comunicación, según se pudo mostrar en una investigación precedente realizada sobre la misma muestra (Laudano et al., 2015). Dicho de otra manera: existe una disposición entre las organizaciones que emplean una red social a sumar o incursionar en el uso de otra.

Concentrado el análisis en los 12 casos relevados, la primera dimensión a considerar trata, por un lado, sobre la referencia o vínculo que establecen las bibliotecas desde su sitio oficial a Twitter y, por otro, de los niveles de descripción institucional empleados en la cuenta como elementos de identificación para aquellos interesados en seguir o contactarse (se trate de usuarios reales, potenciales u ocasionales). En cuanto al primer aspecto - tal y como sucede con el uso de Facebook-, 6 de los 12 casos trabajados no establece ninguna conexión desde la web institucional hacia Twitter (Gráfico 1, 1ra columna). Esto significa que ubicar esas cuentas para los usuarios implica apelar a otras estrategias de localización, ya sea empleando algún buscador o asociándose desde las sugerencias que la propia red social ofrece. Desde el punto de vista organizacional, esta carencia es una prueba de las dificultades bibliotecarias que se extienden en la planificación de los recursos y las políticas comunicacionales. Asimismo, este resultado vuelve a contradecir las sugerencias que la bibliografía especializada ha dado sobre el asunto, lo cual brinda indicios de cierto nivel de experimentación desde la práctica.

El segundo aspecto admite dos puntos de análisis: la descripción escrita y la información visual, ambas cuestiones derivadas del diseño de contenido previsto por la estructura de Twitter. El Gráfico 1 enseña que, con excepción de la referencia que se establece desde Twitter hacia el sitio oficial de la biblioteca (10/12), los demás elementos informativos de carácter general y esencial, como la descripción institucional, los horarios de apertura, los servicios y la locación, son escasamente completados. Lo contrario sucede con la identificación del establecimiento que puede apreciarse mediante el uso de las imágenes de perfil y fondo, que por lo regular ofrecen un efecto de complementariedad. En este sentido, el fondo es ocupado en la mitad de los casos por una fotografía de la biblioteca (6/12) y, de modo marginal, por imágenes alusivas como anaqueles y libros, por algún logotipo o mediante una figura que muestra la adhesión a una causa de orden público, como lo fue la histórica convocatoria y multitudinaria manifestación "Ni una menos" el 3 de junio de 2015 en todo el país, consigna que aglutinó reclamos de lucha en torno a la violencia contra las mujeres. Sólo dos bibliotecas dejaron sin completar este espacio. Los perfiles muestran menor dispersión: en 6 casos se trata de la imagen de la biblioteca, en 5 del logotipo y, en el restante, de una gráfica alusiva al 130 aniversario de la institución. Lo que deja esta descripción es una asociación inmediata entre el ámbito virtual (el 
de la red social) y el espacio físico, una identificación que, en ausencia de los detalles escritos, sólo puede ser apreciada por quienes ya conocen la biblioteca en cuestión, esto es: los usuarios que día a día habitan el lugar.

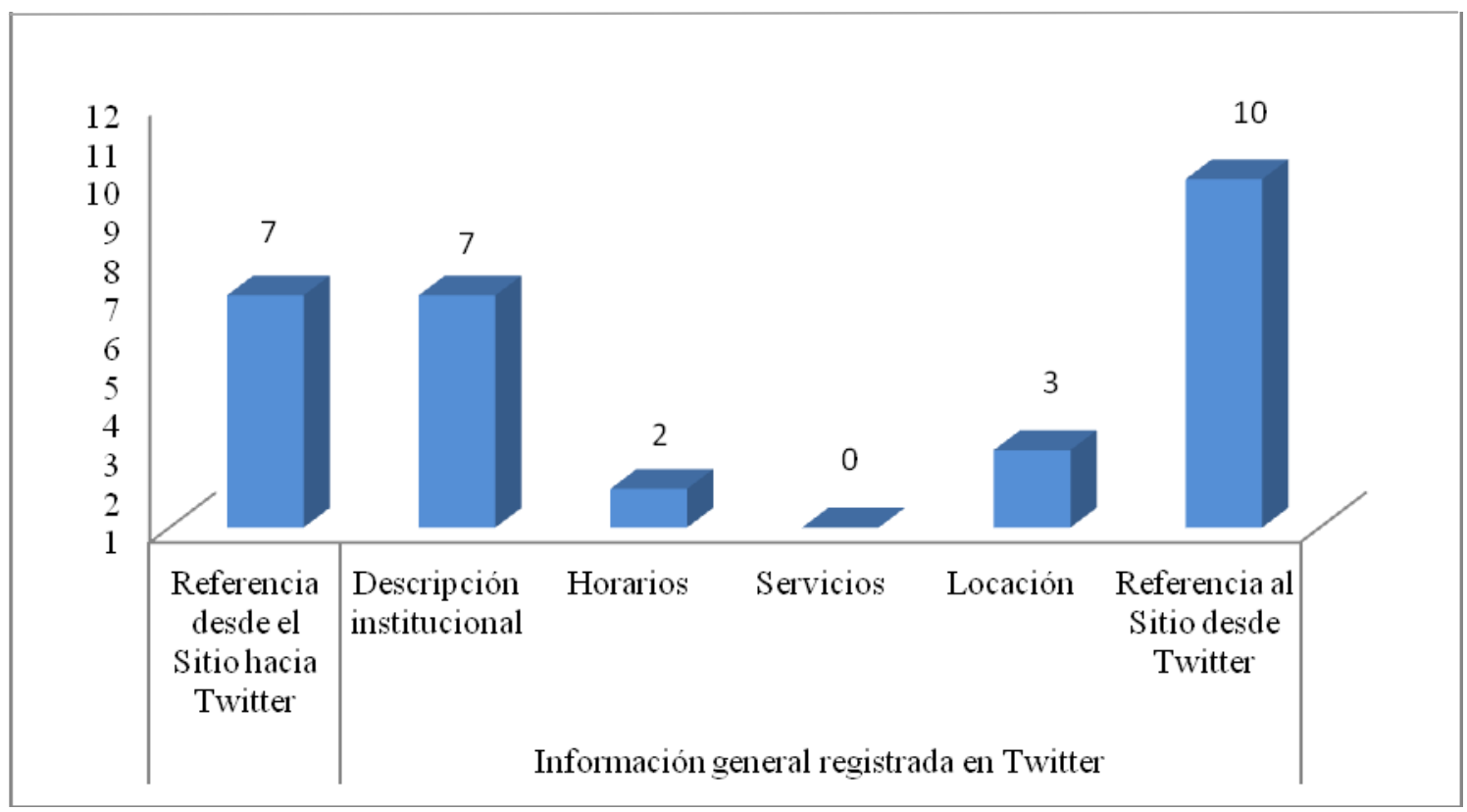

Gráfico 1. Información institucional.

El volumen histórico de tuits (12538), seguidores (8722) y siguiendo (2969) del conjunto de instituciones que forman la muestra no constituye por sí mismo un dato relevante, en tanto que estas ocurrencias se acumulan en el largo de los años. Resulta lógico, por lo tanto, que exista una diferencia sustancial entre las que recién se inician en la actividad y aquellas que llevan en ella un tiempo prudencial. El siguiente gráfico de dispersión enseña tres momentos de arribo al uso de Twitter:

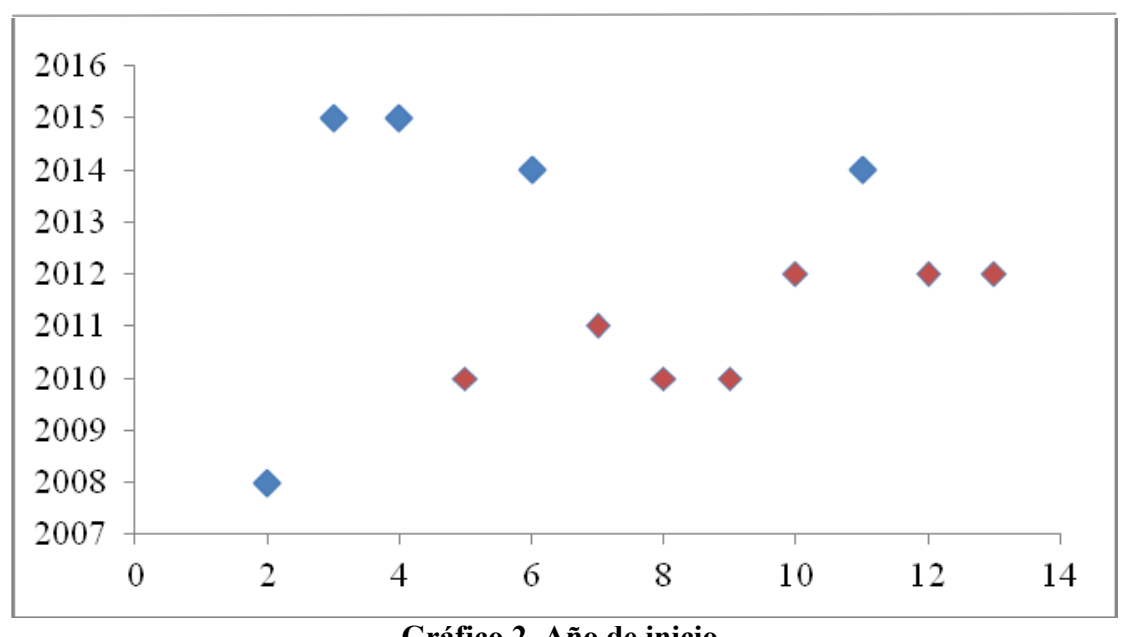

Gráfico 2. Año de inicio.

Llamaremos "Recién llegadas" a las bibliotecas que comenzaron a utilizar Twitter entre 2013 y 2015; "Establecidas", a las que lo hicieron entre 2010 y 2012 y, por último, designaremos como "Caso innovador" a la institución que inició su cuenta en 2008, sin duda un arribo temprano si se considera que Twitter salió al mercado en 2006. Al tomar como referencia estas categorías, resulta previsible que el acumulado promedio de tuits y seguidores difiera sustantivamente entre unas y otras, tal como lo enseña el Gráfico 3: 


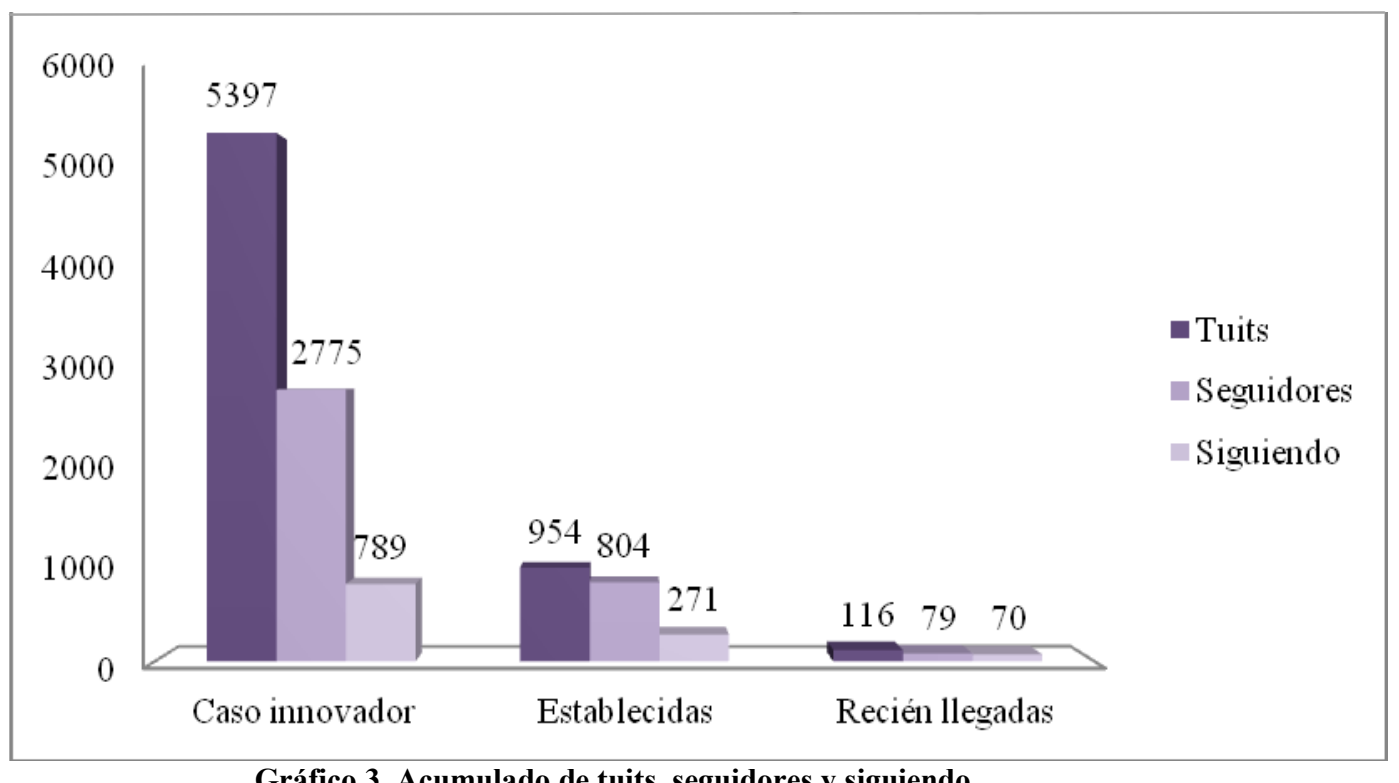

Gráfico 3. Acumulado de tuits, seguidores y siguiendo.

Por otro lado, la gráfica nos devuelve una jugosa constatación al fijar la atención entre el Caso Innovador y las Establecidas: mientras que en el primero hay una diferencia sustancial entre el volumen de tuits y seguidores, las segundas dejan ver una paridad notable (análoga a la que enseña el grupo de Recién llegadas). Esta circunstancia indica una desaceleración en el crecimiento inicial de los seguidores, que sólo aumentará progresivamente conforme ingresen nuevas cohortes de estudiantes a las unidades académicas respectivas. En cuanto a las cuentas seguidas por las instituciones, el desfase no es tan evidente como en las variables precedentes, lo que enseña que el punto de saturación en la elaboración de la red de contactos se alcanza en la primera fase de uso.

Al considerar esas referencias generales, la cuestión es dilucidar si los años de experiencia redundan en comportamientos diferentes al momento de tuitear información. El Gráfico 4 revela este aspecto:

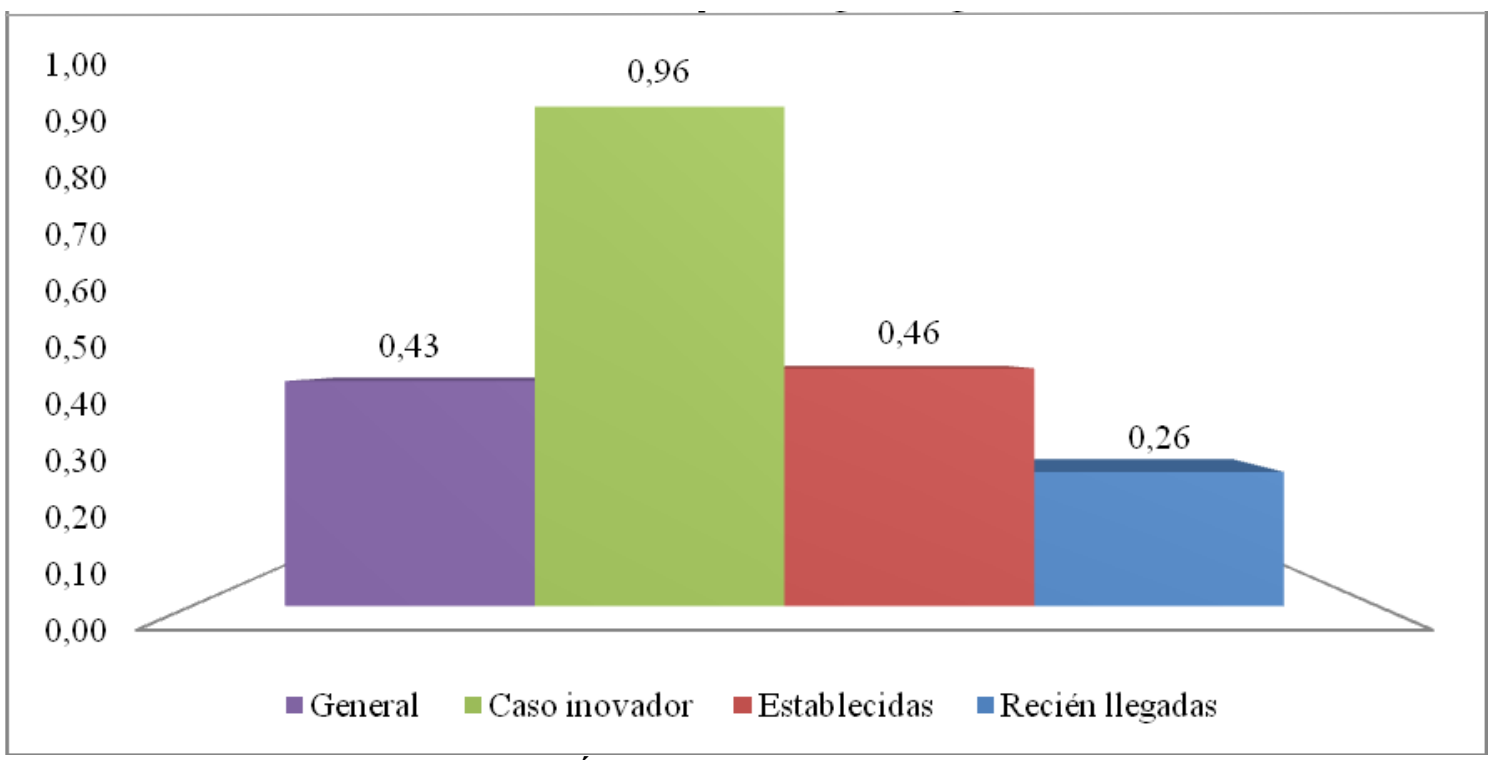

Gráfico 4. Índice de tuits por día según categoría de arribo.

Se puede observar que, mientras el promedio general de tuits por día dentro del período de referencia está en 0,43 (esto es, cerca de un tuit cada dos días) y, en este sentido, el valor se ajusta al comportamiento de la categoría Establecidas $(0,46)$; el Caso Innovador está muy por encima de ese valor $(0,96)$ y las Recién Llegadas $(0,26)$ muy por debajo, esto es: más y menos del doble respectivamente. Esto indica que, en la medida en que las bibliotecas ganan experiencia en el manejo de las redes sociales como una de las modalidades de comunicación, la frecuencia de uso 
fluye con mayor regularidad. Este asunto es, como quedó dicho, un valor imprescindible para mantener cierta fluidez de contacto con la comunidad de seguidores.

Ahora interesa observar los temas que motivan la utilización de Twitter por parte de las bibliotecas. A partir de la bibliografía estudiada (Aharony, 2010; Stvilia y Gibradze, 2014) y de los resultados preliminares arrojados en la prueba piloto, se organizaron 8 clases de tuits: colección (incluye novedades bibliográficas, expurgo, donaciones, repositorios); servicios (refiere a los propiamente bibliotecarios, pero también abarca los avisos de gestión, los cursos de usuarios, las sugerencias sobre herramientas web y las notas periodísticas de interés potencial); institución mayor (concentra las noticias de la universidad, exceptuando los eventos); eventos propios (hace alusión a las jornadas, los talleres, las charlas y los ciclos de cines, entre otra gama de actividades realizadas en la biblioteca, estén organizadas o no por el establecimiento); difusión de eventos (remite a la promoción de propuestas académicas y profesionales de otras instituciones); otras bibliotecas (se compone de información bibliotecaria); efemérides (contiene las reseñas sobre las fechas conmemorativas y los aniversarios); protocolo (comprende los agradecimientos, las salutaciones y los reconocimientos). El rendimiento de cada una de estas categorías se dispuso en relación con las resonancias que tuvieron entre los usuarios. Sobre este aspecto, cabe consignar que los seguidores de las cuentas estudiadas rara vez escriben una respuesta. Por lo tanto, para obtener una idea aproximada de la recepción de los mensajes se tomaron como indicadores los retuis (RT) y los favoritos recibidos (F). El Gráfico 5 muestra, entonces, los resultados en cantidades absolutas de tuits (T), RT y favoritos por cada una de las clases organizadas:

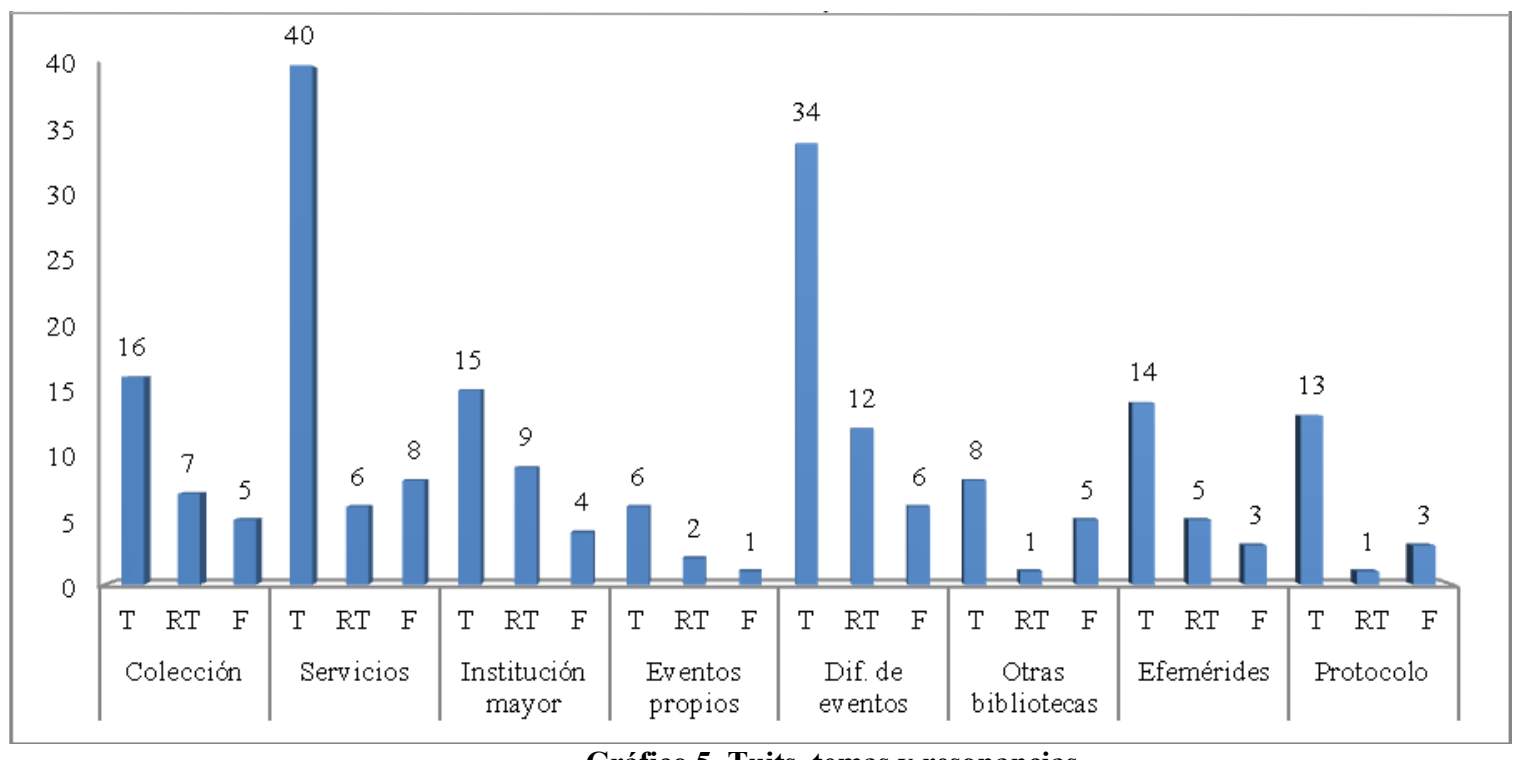

Gráfico 5. Tuits, temas y resonancias.

En relación a los tuits, los servicios encabezan las preferencias de las bibliotecas, llegando al $27 \%$ del total. Igual proporción alcanza la difusión de eventos, si en la ecuación se suman las categorías relativas a la promoción de actividades propias (4\%) y externas (23\%), tal como puede constatarse en el estudio de Stvilia y Gibradze (2014). Este nivel de ocurrencia no resulta sorpresivo: en la vida cotidiana de estas instituciones estos aspectos se actualizan permanentemente. Caso contrario sucede con el resto de los temas, que en términos generales rondan el $10 \%$ (colección, 11\%; efemérides, 10\%; institución mayor, 10\%; protocolo, 9\%; otras bibliotecas, $6 \%$ ).

En relación a las respuestas, el gráfico precedente nos permite ver que hay un desfase importante entre la cantidad de tuits que acumulan las categorías principales y el número de respuestas recibidas en términos de RT y favoritos. Este fenómeno contradice las expectativas puestas sobre el potencial de intercambio biblioteca-usuario augurado para esta herramienta en sus inicios (Mathews, 2008). Para completar esta aproximación fue necesario organizar, no obstante la constatación general, un índice que equilibre la disparidad de las ocurrencias. El Gráfico 6 muestra estas relaciones: 


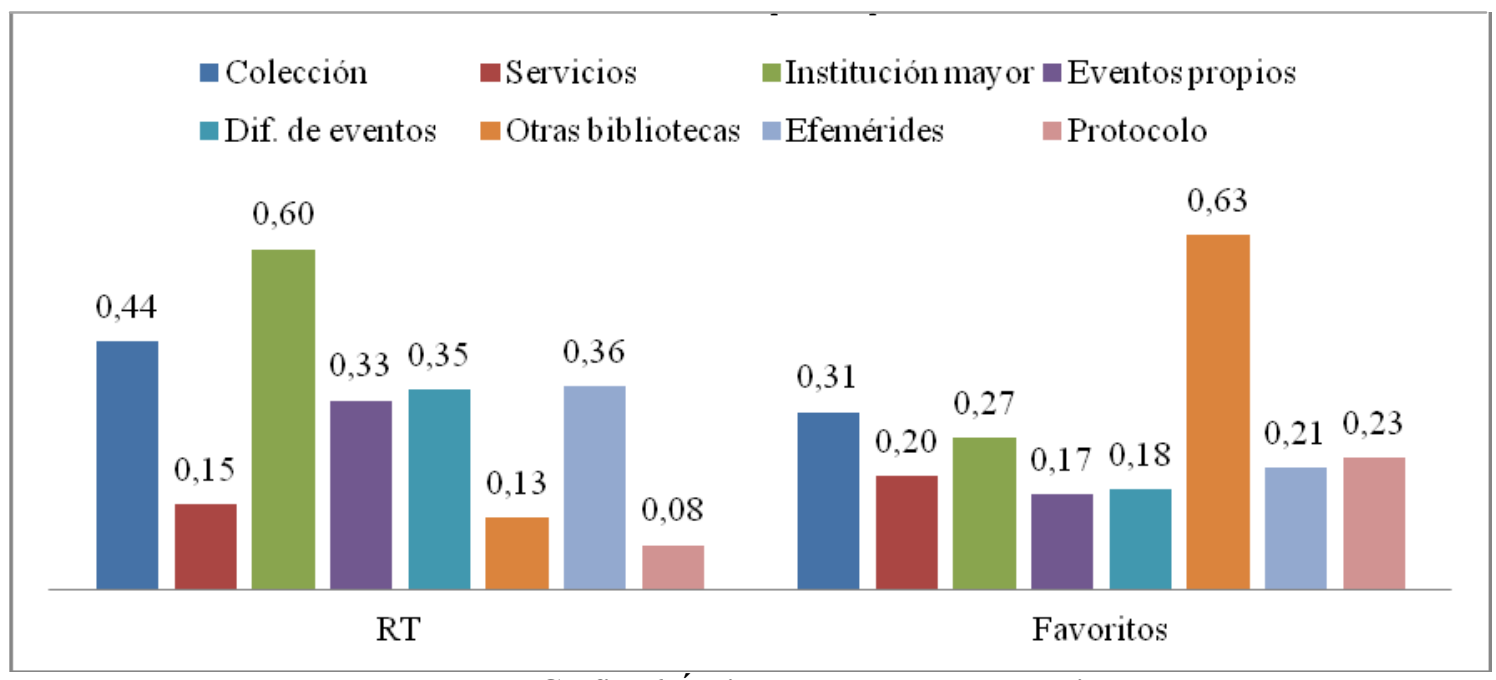

Gráfico 6. Índice de respuesta por temáticas.

La lectura de esta comparativa enseña que, por ejemplo, cada tres tuits sobre la difusión de eventos se recibe un RT, mientras que se necesitan poco más de cinco en la misma categoría para obtener un favorito. Las novedades concernientes al ámbito universitario (institución mayor) parecen ser las más valoradas por los usuarios, que las incorporan en sus propias cuentas - a fin de alertar a sus compañeros o colegas-. Entre los favoritos se destaca la información que corresponde a otras bibliotecas, una cuestión que está más relacionada con la dinámica de trabajo del personal bibliotecario que con el interés de sus lectores.

Anteriormente se mostró el nivel cuantitativo de tuiteo según las categorías de arribo durante el período de referencia que ocupó la muestra. Ahora interesa volver sobre esta segmentación y este marco temporal para analizar los temas escogidos por las bibliotecas y detectar la existencia de dinámicas semejantes y diferenciales. El Gráfico 7 condensa esta información mediante una comparativa entre el promedio de tuits de las bibliotecas en conjunto y los realizados por el Caso innovador, las Establecidas y las Recién llegadas.

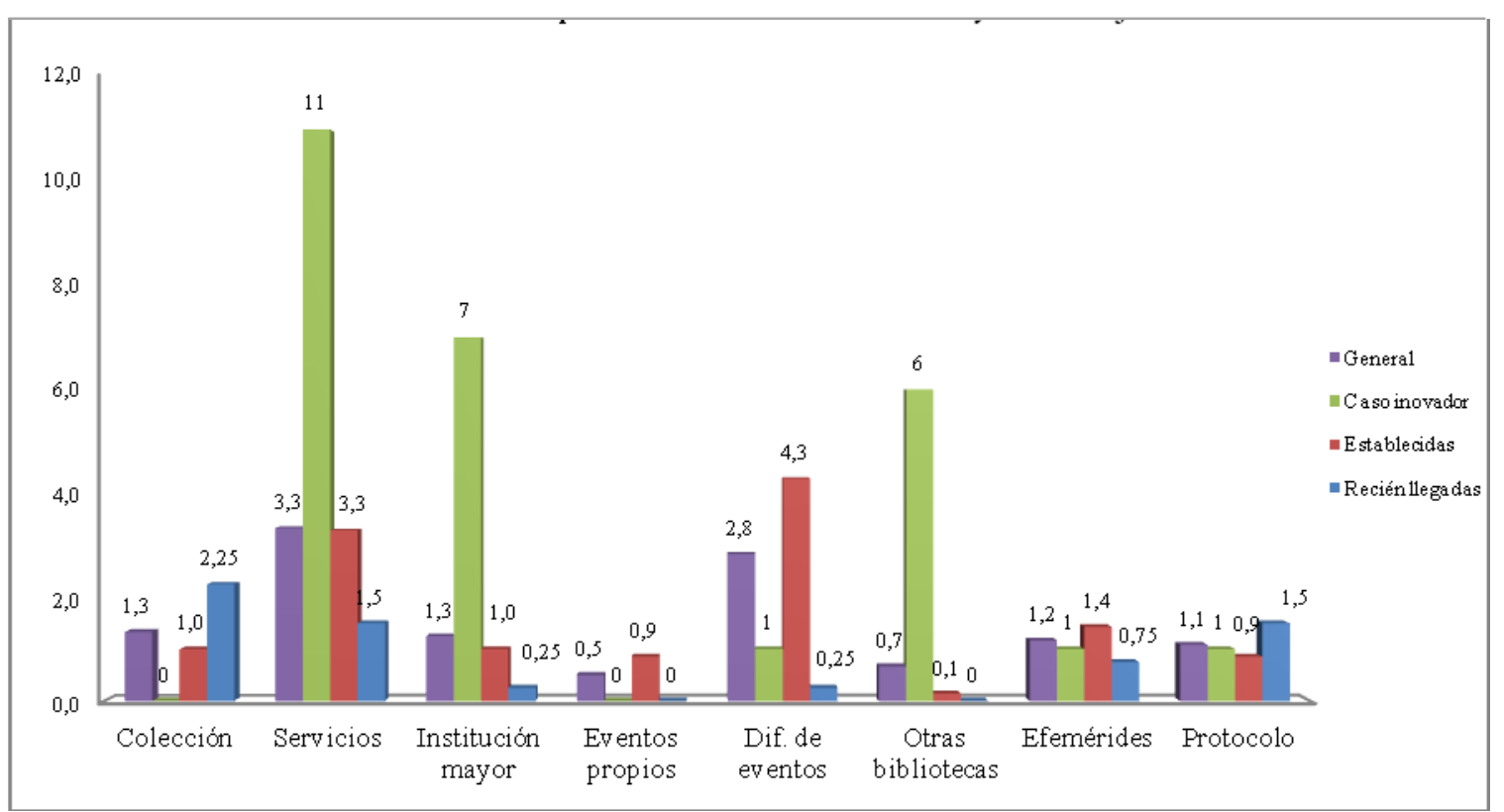

Gráfico 7. Promedio de tuits entre el 1 y el 26 de junio de 2015.

Al momento de seleccionar los temas de tuiteo se observa que cada grupo concentra la atención en dos o tres categorías. Es claro que todas las bibliotecas enfatizan la difusión de los servicios, aspecto que se corresponde con las ocurrencias globales de este género de novedades. Pero mientras el Caso Innovador privilegia simultáneamente la producción de información concerniente a la institución mayor y otras bibliotecas, las Establecidas lo hacen con la 
difusión de eventos y las Recién llegadas con la clase colección. Llama la atención, asimismo, la regularidad de los rubros efemérides y protocolo, cuya frecuencia de aparición es semejante en las cuentas de cada establecimiento.

Además de la elaboración y la publicación de mensajes, Twitter tiene entre sus opciones de manejo la posibilidad de incorporar a la cuenta propia las novedades generadas por terceros mediante un simple paso: el retuit. Esta, sin embargo, no es opción que las bibliotecas utilicen con regularidad: en términos generales entre 1 y 3 RT en 26 días. Solo un caso muestra una particular actividad en este rubro, contabilizando 86 RT entre el 1 y el 26 de junio. El Gráfico 8 compara esta circunstancia, al tiempo que enseña que los RT provienen de cuentas afines a la institución, se trate de la universidad o la unidad académica en cuestión, o de entidades vinculadas con el campo, como gremios, asociaciones, colegios profesionales, etc.

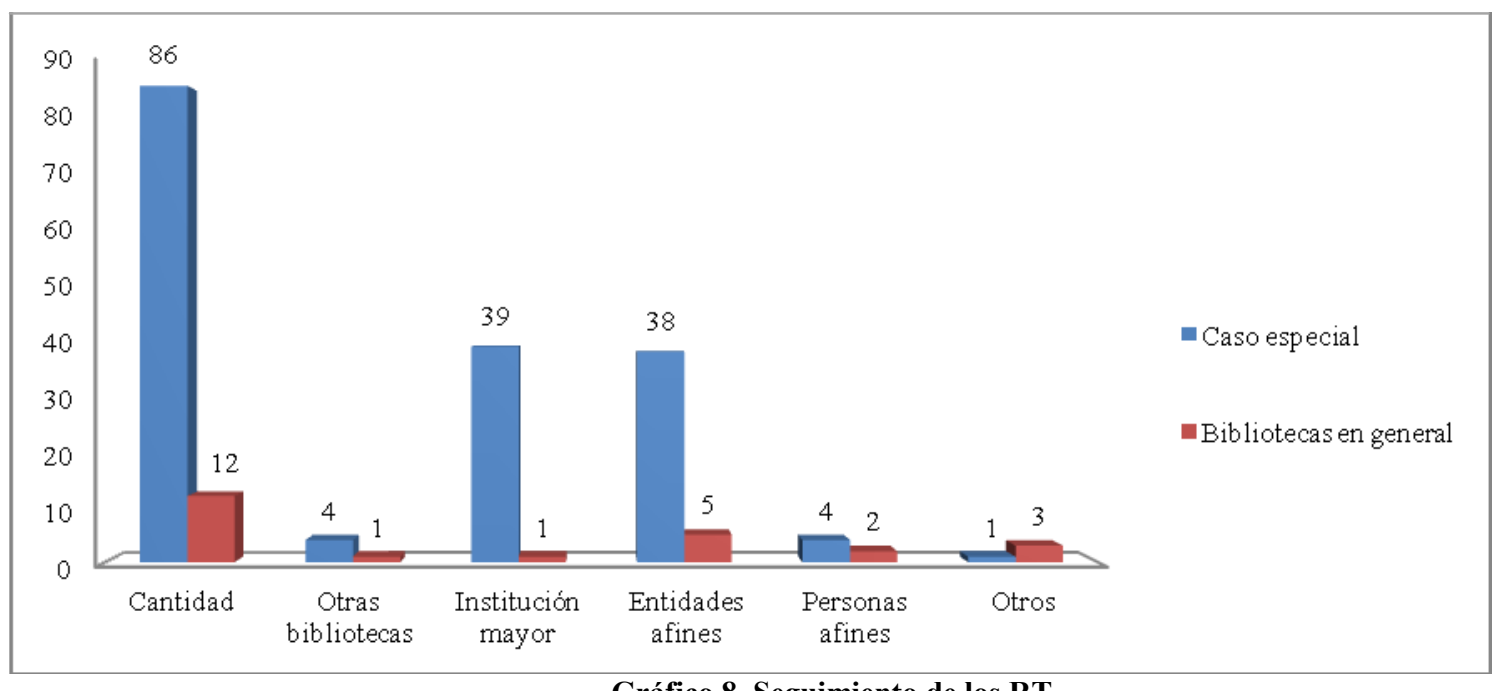

Gráfico 8. Seguimiento de los RT.

\section{CONCLUSIONES}

Si bien en la actualidad Twitter se considera una plataforma relevante dentro de las redes sociales por los niveles de apropiación en distintos sectores de la sociedad argentina; en el ámbito de las bibliotecas universitarias los resultados del relevamiento en 43 instituciones académicas no concuerdan con tal panorama auspicioso, ya que sólo 12 bibliotecas tienen cuenta oficial en funcionamiento. Situación que reafirma la escasa adopción constatada por otros estudios realizados en Argentina.

Si este dato se pone en relación con la fecha de adopción de la plataforma, se observa que sólo un caso puede dar cuenta de una trayectoria sostenida de siete años de uso (desde 2008); mientras que el resto a lo sumo estaría contando con cinco años de puesta a prueba de los beneficios de la red para los fines institucionales. En este sentido, es propicio observar que, si bien es dable contemplar ciertas inconsistencias en el uso al considerar que muchas de las bibliotecas atraviesan un período de aprendizaje, no debe dejar de señalarse que excepcionalmente se siguen las recomendaciones trazadas por la bibliografía especializada. Al respecto, en primer lugar se sugiere contemplar la importancia de planificar una estrategia comunicacional integral, para evaluar de antemano el potencial que facilita cada herramienta, acorde a los objetivos institucionales y las prácticas comunicacionales preponderantes de los usuarios. Dentro de estas consideraciones, una dimensión insoslayable se vincula con la dedicación temporal y de personal que insume el sostenimiento de la cuenta, ya que un uso superficial o sin la dedicación necesaria podría no ser beneficioso para la imagen institucional. Respecto de los usos puntuales de la cuenta, para potenciar su llegada, ciertas directrices destacan acciones tales como arrobar a seguidores, disponer de listas de envíos y/o construir hashtags, tanto como recabar información de necesidades e intereses de seguidores de la cuenta de manera periódica.

En términos generales, se extiende una necesidad de reflexionar acerca de la construcción de la voz pública de la biblioteca, así como los alcances que pueden generar las controversias en línea por el potencial de la viralización, las posibilidades del trabajo en red (y/o junto a otras) y la construcción de comunidad, entre otros aspectos. Asimismo, cabe recordar que en la mayoría de los casos esta plataforma no opera con exclusividad sino de manera simultánea con otras herramientas, entre ellas, en primer lugar, Facebook, la otra red social que se ha popularizado en el país. Con todo, aún se puede caracterizar el estadio de las relaciones entre las bibliotecas universitarias y las redes sociales como 
experimental, pese a los años transcurridos desde la irrupción de estas modalidades de comunicación. En este sentido, resulta probable que, en la búsqueda de estar en el lugar de los usuarios, las bibliotecas incorporen estos nuevos canales de comunicación sin un análisis previo y, por consiguiente, sin establecer una política comunicacional acorde a las necesidades informativas y a los intereses de sus propios lectores y lectoras. El resultado de esta implementación repite las incongruencias descriptas por otras investigaciones en diferentes latitudes. Y, lo que resulta todavía más preocupante, el nivel de respuesta generado entre los usuarios es notablemente bajo, en buena medida porque las estrategias actuales de las bibliotecas se asientan sobre las lógicas comunicacionales impresas en lugar de apelar a prácticas más acordes con los uso que los usuarios le brindan habitualmente a estos medios.

Una constatación semejante sugiere una serie de cuestiones a indagar a través de otras estrategias metodológicas de corte cualitativo. Así, por ejemplo, la organización de entrevistas con el personal bibliotecario podría brindar un panorama de las condiciones materiales y laborales bajo las cuales son utilizadas las redes sociales en general, y Twitter en particular. De esta manera, sería posible aproximarse a los porqués y los cómos de problemas relativos a los motivos que subyacen a la elección de una red por otra, a los usos simultáneos de las plataformas disponibles, a las políticas comunicacionales que se siguen (si es que existen), a la planificación de estudios de usuarios que contribuyan con la formación de un conocimiento del campo, al tiempo real dedicado a la comunicación institucional, a la formación del personal de la biblioteca en asuntos de esta índole, a las posibles restricciones o limitaciones por parte de las autoridades y, en general, al espacio que los bibliotecarios le asignan a Twitter y a las demás redes sociales entre sus propias prácticas y perspectivas profesionales. Un paso más allá queda el análisis de estos aspectos en la intersección con las expectativas que la comunidad de lectores manifiesta en relación a la biblioteca y sus intervenciones.

\section{BIBLIOGRAFÍA}

AHARONY, N. Twitter Use in Libraries: An Exploratory Analysis. Journal of Web Librarianship, 2010, vol. 4, nº 4, p. 333-350.

ALVIM, L. Da blogosfera ao Facebook: o paradigma da comunicação nas bibliotecas portuguesas. Cadernos de bibiblioteconomia, arquivistica e documentacao, 2009/2010, p. 29-59.

BODNAR, J. y DOSHI, A. Asking the right questions: a critique of Facebook, social media, and libraries. Public services quaterly, 2011, vol. 7, $\mathrm{n}^{\mathrm{o}} 3$, p. 102-110.

CAVANAGH, M. Micro-blogging practices in Canadian public libraries: A national snapshot. Journal of Librarianship and Information Science, 2014, p. 1-13.

CHEN, D.Y.T.; CHU, S.K.W. y XU, S.K. How do libraries use social networking sites to interact with users. ASIS\&T Annual Meeting, 2012, 28 al 31 de octubre de 2012. [en línea]. Disponible en: $<$ https://www.asis.org/asist2012/proceedings/Submissions/85.pdf $>$. [Consulta: 10 de marzo de 2015].

DEL BOSQUE, D.; LEIF, S.A. y SKARL, S. Libraries atwitter: Trends in academic library tweeting. Reference Services Review, 2012, vol. 40, n 2, p. 199-213.

DE FRANCESCHI, A. \#Biblioteca \#Usuarios: Twitter en bibliotecas universitarias argentinas: la interacción con los usuarios. 2014. [en línea] Disponible en: <http://eprints.rclis.org/24008/>. [Consulta: 20 de febrero de 2015].

DE VOLDER, C.; GONZÁLEZ TERÁN, Y. y GUTIÉRREZ, F.G. Microblogging: el caso Twitter en bibliotecas universitarias argentinas [en línea]. Disponible en: $<$ http://eprints.rclis.org/18020/>. [Consulta: 25 de febrero de 2015].

FIELDS, E. A unique Twitter use for reference services. Library Hi Tech News, 2010, vol. 27, nº 6-7, p. 14-15.

FORRESTAL, V. Making Twitter Work: A Guide for the Uninitiated, the Skeptical, and the Pragmatic. The Reference Librarian, 2011, vol. 52, $\mathrm{n}^{\circ} 1-2$, p. 146-151.

HAGMAN, J. Joining the Twitter Conversation. Public Services Quarterly, 2012, vol. 8, nº 1, p. 78-85.

LAUDANO, C.N.; CORDA, M.C.; PLANAS, J. y KESSLER, M.I. Los usos de la red social Facebook en las bibliotecas de institutos y centros de investigación en Argentina. Palabra Clave (La Plata), 2014, vol. 4, $\mathrm{n}^{\circ} 1, \mathrm{p}$. 20-32. [en línea]. Disponible en: <http://www.palabraclave.fahce.unlp.edu.ar/article/view/PCv4n1a02/6174>. [Consulta: 15 de marzo de 2015].

LAUDANO, C.N.; CORDA, M.C.; PLANAS, J. y KESSLER, M.I. Los usos de la red social Facebook por parte de bibliotecas universitarias argentinas. Reflexiones en torno a las dinámicas comunicativas en la Web 2.0. Revista Interamericana de Bibliotecología. En prensa.

MARGAIX ARNAL, D. Las bibliotecas universitarias y Facebook: cómo y por qué estar presentes. El profesional de la información, 2008, vol. 17, $\mathrm{n}^{\circ}$ 6, p. 589-601.

MATHEWS, B. Twitter and the Library: Thoughts on the Syndicated Lifestyle. Journal of Web Librarianship, 2008, vol. 2 , no 4 , p. 589-593. 
SEWELL, R.R. Who is following us? Data mining a library's Twitter followers. Library Hi Tech, 2013, vol. 31, nº 1, p. 160-170. [en línea]. Disponible en: <http://dx.doi.org/10.1108/07378831311303994>. [Consulta: 18 de marzo de 2015].

STUART, D. What are libraries doing on Twitter? Online (Weston, CT), 2010, vol. 34, nº 1, p. 45.

STVILIA B. y GIBRADZE, L. What do academic libraries tweet about, and what makes a library tweet useful? Library \& Information Science Research, 2014, vol. 36, $\mathrm{n}^{\mathrm{o}} 3-4, \mathrm{p} .136-141$.

VERISHAGEN, N. y HANK, C. Are there birds in the library? The extent of Twitter adoption and use by Canadian academic libraries, First Monday, 2014, vol. 19, $\mathrm{n}^{\circ}$ 11. [en línea]. Disponible en: $<$ http://firstmonday.org/ojs/index.php/fm/article/view/4945/4161>. [Consulta: 20 de marzo de 2015].

WADDELL, D.; BARNES, M. y KHAN-KERNAHAN, S. Tapping into the Power of Twitter: A Look at Its Potential in Canadian Health Libraries. Partnership: The Canadian Journal of Library and Information Practice and Research, 2012, vol. 7, $\mathrm{n}^{\circ}$ 2, p. 1-12.

WAN, G. How academic libraries reach users on Facebook. College \& undergraduate libraries, vol. 18, n 4, p. 307318. 